\title{
Solid-state dewetting with a magic thickness: Electronic dewetting
}

\author{
Anna Chame ${ }^{1}$ and Olivier Pierre-Louis ${ }^{2}$ \\ ${ }^{1}$ Instituto de Física, Universidade Federal Fluminense, Avenida Litorânea s/n, 24210-340 Niterói RJ, Brazil \\ ${ }^{2}$ Institut Lumière Matière, UMR5306 Université Lyon 1-CNRS, Université de Lyon 69622 Villeurbanne, France
}

(Received 3 June 2014; revised manuscript received 18 September 2014; published 7 November 2014)

\begin{abstract}
We investigate the dewetting dynamics of ultrathin solid metal films. In these films, quantum confinement of electrons is known to produce a complex wetting potential, leading to "magic thicknesses" which are strongly favored energetically. We introduce a kinetic Monte Carlo model which accounts for a magic thickness. When the driving forces are small enough, the dewetting proceeds from the edges of the films and we find two regimes: (i) Regime I: for stronger driving forces, no dewetting rim is formed along the edge of the film, and magic-height fingers perpendicular to the film edge invade the film. (ii) Regime II: for smaller driving forces, magic-height fingers form parallel to the edge of the film. In this regime, a magic-height dewetting rim forms, and subsequently breaks up due to the emergence of two instabilities: rim closure failing, and layer-by-layer nucleation of holes in the film behind the rim. In both regimes, the dewetting velocity and the typical wavelength observed in the simulations are found to be in good agreement with a maximum velocity principle based on a simple model accounting for diffusion limited dynamics. Finally, the labyrinthine morphology resulting from the dewetting process and its typical length scales are found to be consistent with experimental observations. For stronger driving forces, we observe homogeneous nucleation. Depending on the relative values of the magic height and the film height, the dewetting process starts with the formation of holes or with the formation of magic-height islands.
\end{abstract}

DOI: 10.1103/PhysRevB.90.195408

PACS number(s): 81.10.Aj, 05.70.Ln, 68.55.-a

\section{INTRODUCTION}

In the past 15 years, solid-state dewetting has been the subject of many experiments [1-16] and models. While early theories were based on macroscopic continuum descriptions [17-19], recent models accounting for the atomistic structure of crystalline films have pointed out the crucial role of anisotropy (including singular facets and the related dynamics of atomic steps) on the dewetting dynamics of thin films [20-22]. The downsizing of film thicknesses up to the nanoscale raises the question of the dewetting dynamics of films consisting of a few atomic layers.

Here, we wish to focus on a phenomenon which appears for nanometrically thin metallic films: the quantum confinement of the conduction electrons in a thin metallic film, which induces thickness dependent properties such as oscillations in the work function [23] or in the film surface energy [24]. The complex wetting potential produced by the combination of these quantum size effects and the atomic discreteness of the film thickness lead to the so-called magic thicknesses, which are strongly favored energetically. This phenomena is known to have a strong impact on the morphology of islands and films formed during growth, as discussed in theory [23,25,26] and experiments [27-32] for various metals.

Here, we aim to describe the effects of magic thicknesses on the dewetting dynamics of thin films. In experiment, such as, e.g., for $\mathrm{Ag} / \mathrm{Si}(111)$ [2,29], growth and annealing are not necessarily well separated, and their respective influences on the final morphology are often mixed. Even if flat films can be grown at low enough temperatures when the morphology is controlled by kinetics, these films may destabilize under annealing to gain energy via a specific dewetting process. Here, we wish to describe the main features of this dewetting process using a simplified wetting potential which keeps the main physical ingredient: the existence of a magic thickness. Since the growth of thin films in the presence of magic film thicknesses was called "electronic growth" in the literature [25], we denote the related dewetting process as "electronic dewetting." We find some specific features of the electronic dewetting process which can help experimentalists to distinguish it from usual dewetting. These features include not only the expected flat-top islands with a magic thickness, but also the absence of stable dewetting rims, the possibility of forming fingers parallel or perpendicular to the dewetting front when dewetting is initiated at the edge of a film, and the possibility of forming magic islands first or holes first during homogeneous nucleation far from the film edges. We also provide a quantitative expression for the width of the fingers, which can be compared to experiments.

In Sec. II, we present our kinetic Monte Carlo (KMC) model with wetting properties and a magic film thickness. In Sec. III, we present the results of the simulations for the dewetting process starting from an initially straight film edge. We focus on the cases of small or moderate driving forces, when homogeneous nucleation is absent, and find two distinct regimes. In Regime I, for strong enough driving forces, no rim is formed along the dewetting front, and magic-height fingers appear from the first stages of the dewetting process. In Regime II, for weaker driving forces, a dewetting rim is formed along the edge of the film. However, as opposed to the case where no magic thickness is present [21], the dewetting rim is always unstable. We observe two pathways for the destabilization of the dewetting rim in regime II. The first one is layer-by-layer nucleation of holes behind the dewetting rim. The second one is the incomplete closure of the magic-height rim, leading to gaps through which dewetting proceeds. The different dynamics of regime I and regime II leads to very different morphologies at least in the initial stages of dewetting, with fingers roughly perpendicular or parallel to the dewetting front, respectively. 
For both regime I and II, the length scale of the resulting magic-height finger structures and the speed of the dewetting fronts are found to be in good agreement with a model based on a simple maximum velocity principle. In Sec. IV, we focus on homogeneous nucleation away from the film edge. We point out the crucial role of the relative values of the initial film thickness and of the magic thickness in order to observe homogeneous nucleation, and to observe percolating or nonpercolating networks for magic-height islands. During homogeneous dewetting the denuded substrate area increases as $t^{3}$, a behavior which is similar to that of monolayer dewetting [33], and different from the approximate scaling of multilayer ultrathin films $\sim t^{2}$ in the absence of a magic height $[20,34]$.

We compare our results to experimental observations in Sec. V and show that our results are consistent with the morphologies and with the finger widths observed in experiments. Finally, we summarize our results in Sec. VI.

\section{KMC MODEL WITH A MAGIC THICKNESS}

Our solid-on-solid KMC model (Fig. 1) accounts simultaneously for wetting properties and for a magic thickness. We use a square lattice with periodic boundary conditions. On each site of the lattice, the surface height has an integer value $z \geqslant 0$. The substrate is flat and frozen: substrate atoms at $z=0$ cannot move. Atoms at the surface of the film with $z \geqslant 1$ can hop to nearest neighbor sites with rates

$$
\begin{gathered}
z \neq 1 \text { and } z \neq h_{*} \quad v_{n}=v \mathrm{e}^{-\left(n J+J_{0}\right) / T}, \\
z=1 \quad r_{n}=v \mathrm{e}^{-\left(n J+J_{0}-E_{S}\right) / T}, \\
z=h_{*} \quad r_{n}^{*}=v \mathrm{e}^{-\left(n J+J_{0}-E_{*}\right) / T},
\end{gathered}
$$

where $n$ is the number of neighbors in the same plane, $T$ is the temperature in units of $k_{B}=1, h_{*}>1$ is the magic height, and $J, E_{S}, E_{*}$ are energy parameters.

The rates given above can be related to a simple underlying energetic picture. Atoms in the film with $z \geqslant 2$ and $z \neq h_{*}$ interact with first neighbors in the same horizontal plane via bonds of energy $J$, and with the neighbor just below via vertical bonds of energy $J_{0}$. The energy cost to remove a surface atom with $z \geqslant 2$ and $z \neq h_{*}$ is therefore $\Delta E=$ $n J+J_{0}$. If the neighbor just below the atom belongs to the substrate, i.e., $z=1$, the energy cost for removing an atom is $\Delta E=n J+J_{0}-E_{S}$. Note that $E_{S}$ not only accounts for the change of the vertical bond under a film atom when it is

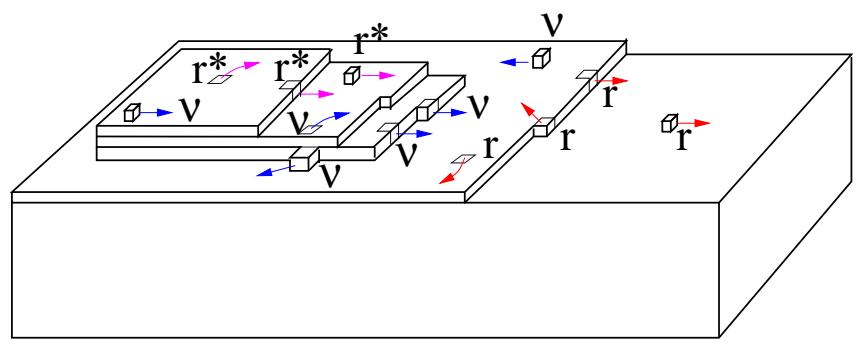

FIG. 1. (Color online) Schematics of the solid-on-solid kinetic Monte Carlo (KMC) model, with hopping rates $v_{n}, r_{n}$, and $r_{n}^{*}$ in the case $h_{*}=4$. in contact with the substrate, but also for the formation of a broken substrate bond. Following this line and within a low temperature approximation [35], $E_{S}$ can be shown to be equal to minus the spreading coefficient [36] used in the context of wetting of liquids. Similarly, the cost for removing an atom at $z=h_{*}$ is $\Delta E=n J+J_{0}-E_{*}$. The energy landscape experienced by a solid film can be summarized in the wetting potential $W(z)$. The wetting potential $W(z)$ of our model can be defined as the difference between the free energy of the film surface at thickness $z$ and the free energy of the film surface for large film thicknesses. Once again within a low temperature picture, where the surface free energies are essentially equal to surface energies, we have $W(z \geqslant 1)=E_{S} \delta_{z, 1}+E_{*} \delta_{z, h_{*}}$. As a remark, in order to account for a magic height at $z=h_{*}$, the energy parameter $E_{*}$ will always be negative in the following.

Using a physical picture where atoms break all bonds in order to diffuse, the hopping rates are then defined as $v \exp [-\Delta E / T]$, where $v$ is an attempt frequency. In order to simplify the notations, we define $v_{0}=v \exp \left[-J_{0} / T\right]$. In the following, we use reduced units: We choose $a, v_{0}$, and $J$ as the length, time, and energy units, respectively. This is formally equivalent to setting $a=1, v_{0}=1$, and $J=1$.

\section{DEWETTING FROM A STRAIGHT FILM EDGE}

\section{A. KMC results}

We start with the analysis of the case of a film which has initially a uniform thickness $z=h$, except for a straight trench along the (100) axis, where the substrate is denuded, i.e., $z=0$. From this initial condition, with $E_{S}>0$ and $E_{*}<0$, we observe two dewetting fronts moving in opposite directions. However, if the driving forces $E_{S}$ and $\left|E_{*}\right|$ are too large, homogeneous nucleation of holes is seen in the film far from the film edge. Here, we postpone the discussion of nucleation to Sec. IV, and we focus on the case of small enough driving forces when no homogeneous nucleation is observed.

In the absence of a magic height, i.e., for $E_{*}=0$, straight dewetting fronts along the (100) orientation are stable in the sense that they exhibit a straight and continuous dewetting rim along the dewetting front, as discussed in Refs. [20,21]. However, in the presence of a magic height, i.e., for $E_{*} \neq 0$, we observe that the dewetting process starting from (100) film edges is always unstable: The final state is composed of fingers with the magic thickness $h_{*}$.

Let us describe the simulation results in more details. The dewetting process starts with the nucleation of twodimensional islands on the top of the film in the vicinity of the film edge. When the driving forces $E_{S}$ and $\left|E_{*}\right|$ are small, these islands merge before the nucleation of another layer above them. In the opposite limit of strong driving forces, magic islands are nucleated before the islands merge. Hence, separated magic islands are formed along the dewetting front. This leads to the two main regimes observed in the simulations, as shown in Fig. 2.

The first regime, hereafter denoted as regime I, and shown in Figs. 2(a) and 2(b), corresponds to the case of strong driving forces. In this regime, no rim is formed along the edge of the film. Magic-height fingers appear, and are left behind the front 

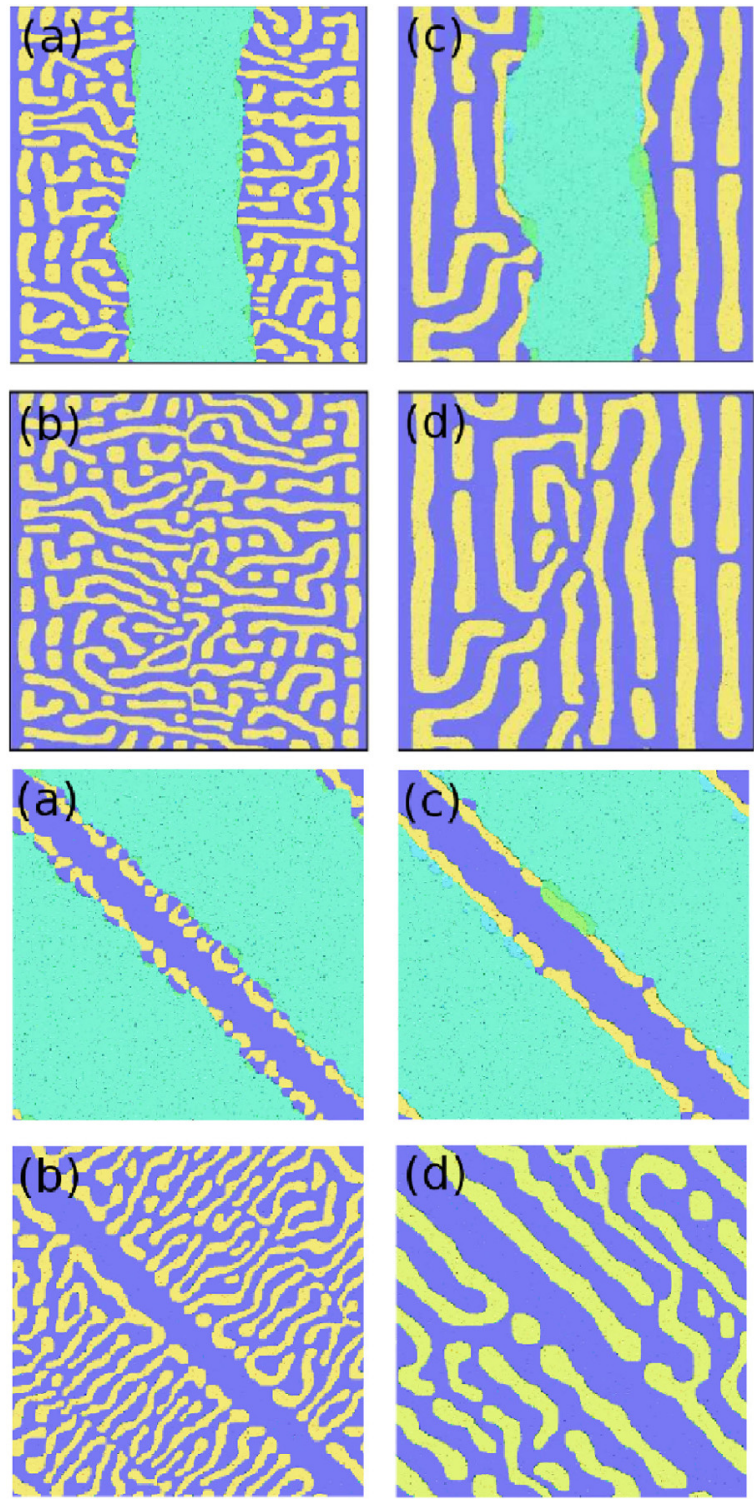

FIG. 2. (Color online) KMC simulations of dewetting in regimes I and II. The blue-yellow (dark-light) scale indicates the local height $z$. Dewetting initiated at a straight film edge in the $800 \times 800$ system with $T=0.4, h=3$, and $h_{*}=7$. Upper panels: initial film edge along (100). Lower panels: initial film edge along (110). (a),(b) Regime I for stronger driving forces with $E_{S}=0.7$ and $E_{*}=-1.0$. (c),(d) Regime II for smaller driving forces with $E_{S}=0.5$ and $E_{*}=-0.5$.

as a result of the dewetting process. These fingers are mainly oriented perpendicularly to the dewetting front.

The second regime, denoted as regime II and shown in Figs. 2(c) and 2(d), is obtained for small driving forces. In this regime, after the initial formation of a continuous rim of height smaller than $h_{*}$ along the film edge, magic-height islands form on the top of the rim. These magic-height islands expand, invading the dewetting rim. But when two magic-height zones meet, they do not always merge, and often leave a gap. Then, the dewetting proceeds beyond the rim through this gap. Such an incomplete closure of the rims was not observed in the absence of a magic thickness [20].

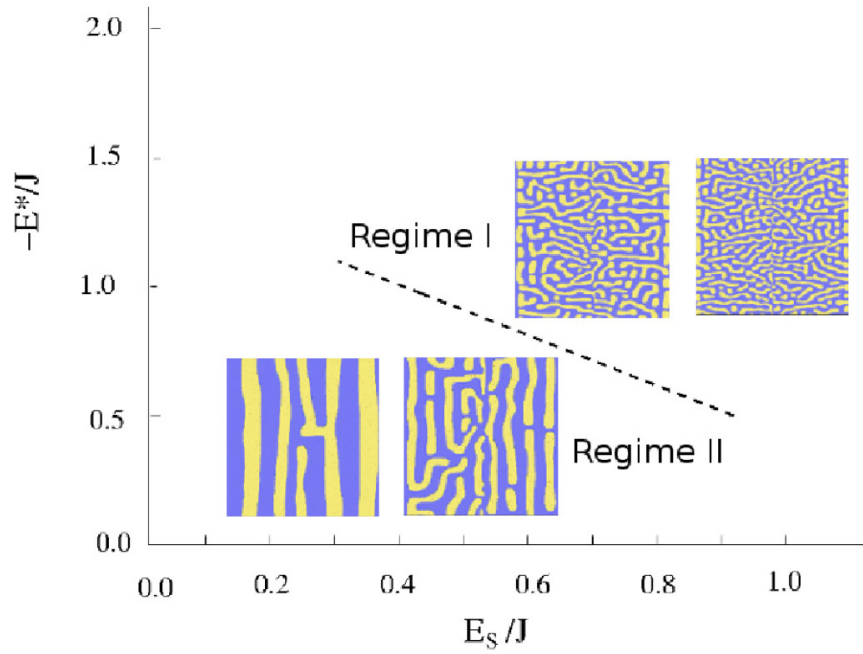

FIG. 3. (Color online) Morphology diagram in the $\left(E_{*} / J, E_{S} / J\right)$ plane. Dewetting started from straight (100) fronts in systems of size $800 \times 800$ with $T=0.4, h=3$, and $h_{*}=7$. The images show the morphology of the film after the dewetting process, when there are only magical height islands left on the substrate. They are centered on the corresponding values of $\left(E_{S}, E *\right)$ : for regime $\mathrm{I},\left(E_{S}, E_{*}\right)=$ $(0.7,-1.0)$ and $\left(E_{S}, E_{*}\right)=(1.0,-1.0)$ and for regime II, $\left(E_{S}, E_{*}\right)=$ $(0.3,-0.5)$ and $\left(E_{S}, E_{*}\right)=(0.5,-0.5)$.

In regime II, we also observe the formation of holes behind the magic-height rims. A similar formation of holes behind the magic-height islands is observed in regime I, but is less frequent, maybe because there is no extended rim. In regime II, these holes grow along the magic-height rims, often leading to the total detachment of the rim from the film. This process may occur periodically leading to magic-height islands mainly oriented parallel to the original front, as seen in Figs. 2(c) and $2(\mathrm{~d})$. This regime could be a transient and the structure of the magic fingers in regime II could be disordered at long times. The limited size of our simulations does not allow us to reach a definitive conclusion on this point.

In a previous paper [21], it was pointed out that the stability of dewetting fronts depends on the front orientation in the absence of a magic height. Indeed, while (100) fronts where found to be stable, (110) fronts are unstable and spontaneously break up into an array of fingers. We find that the situation is very different in the presence of a magic height. Simulations starting from a diagonal trench along the (110) direction actually show the same behavior as those starting along the (100) direction. Indeed, simulations in the lower panel of Fig. 2 show that regime I or regime II is recovered for parameters similar to those found when starting for (100) fronts. These results suggest that, within the regime of parameters studied here, the instability triggered by the presence of a magic height is faster than the instability identified previously for (110) dewetting fronts. The parameter regions in which regimes I and II are observed, are reported in Fig. 3.

\section{B. Layer by layer hole formation behind the rim}

One specific feature that we have observed in KMC simulations when $E_{*} \neq 0$ is the nucleation of holes behind 
the rim. This observation can be explained by the enhanced formation of shallow holes of depth $h_{0}<h$ behind the rim, which play the role of precursors for the formation of a complete hole of depth $h$ (with denuded substrate at its bottom). Indeed, consider the formation of a shallow circular hole with radius $R_{0}$ and depth $h_{0}<h$. The atoms withdrawn to form the hole are transferred to a neighboring magic height rim. The related Gibbs free energy variation reads:

$$
\Delta E=2 \gamma_{0} \pi R_{0}+A_{*} E_{*},
$$

where $\gamma_{0}$ is the free energy of an edge of height $h_{0}$, and $A_{*}$ is the additional area of the magic-height rim. We then use mass conservation

$$
A_{*}\left(h_{*}-h\right)=\pi R_{0}^{2} h_{0},
$$

and find a critical radius $R_{0 c}$ and nucleation barrier $E_{0 c}$ as

$$
\begin{gathered}
R_{0 c}=\frac{\gamma_{0}}{h_{0}} \frac{h_{*}-h}{-E_{*}}, \\
E_{0 c}=\pi\left(\frac{\gamma_{0}}{h_{0}}\right)^{2} \frac{h_{0}\left(h_{*}-h\right)}{-E_{*}} .
\end{gathered}
$$

Let us now consider the direct formation of a full hole of depth $h$ in a film of thickness $h$. Once again, the mass is transferred to a rim of height $h_{*}$. The free energy now reads:

$$
\Delta E=2 \gamma \pi R+A_{*}\left(E_{*}-E_{S}\right),
$$

where $\gamma$ is the free energy of a film of thickness $h$. Using again the mass conservation relation (5), we now find a critical radius $R_{c}$ and the associated nucleation barrier $E_{c}$ as

$$
\begin{gathered}
R_{c}=\frac{\gamma}{h} \frac{h_{*}-h}{E_{S}-E_{*}}, \\
E_{c}=\pi\left(\frac{\gamma}{h}\right)^{2} \frac{h\left(h_{*}-h\right)}{E_{S}-E_{*}} .
\end{gathered}
$$

We shall now use Eqs. (6), (7), (9), and (10) to discuss quantitatively the hole nucleation process. We have previously shown [33] that $\gamma_{\text {step }} \approx 0.42$ for our SOS model at $T=0.4$. We may use this result to evaluate the nucleation barriers in a scenario where the hole is formed by means of the successive nucleation of monolayer holes. In the following, we shall denote this process as layer-by-layer hole formation. We use typical parameter values used in the simulations: $h=3$, $h_{*}=7, E_{S}=0.5, E_{*}=-0.5$, and $T=0.4$. For the formation of a monolayer hole behind the rim, we find from Eqs. (6) and (7): $R_{0 c} \approx 3.4$ and $E_{0 c} \approx 4.5$, leading to an activation probability $\exp \left[-E_{0 c} / T\right] \sim 10^{-5}$. Once this monolayer hole is formed, this hole can expand laterally leaving a film of height $h=2$ adjacent to the magic-height dewetting rim. On this thinner film inside the monolayer hole, another monolayer hole can be formed. Using the same parameters as above except for $h=2$, we now find $R_{0 c} \approx 4.5$ and $E_{0 c} \approx 5.5$, and an activation probability $\exp \left[-E_{0 c} / T\right] \sim 10^{-6}$. The monolayer hole again expands, leaving a monolayer-thick film near the magic-height dewetting rim. The rate of nucleation of a hole in the last monolayer is then increased by the term $E_{S}$, and we obtain from Eqs. (9) and (10) $R_{0 c} \approx 2.5$ and $E_{0 c} \approx 3.3$, with an activation probability $\exp \left[-E_{0 c} / T\right] \sim 2 \times 10^{-4}$. The whole process is therefore limited by the lowest activation probability $\sim 10^{-6}$.

In order to provide a quantitative account of the direct nucleation of a hole with edges higher than one atomic step, we need to evaluate the quantity $\gamma / h$ or $\gamma_{0} / h_{0}$ which enters in the expressions of the nucleation radii and nucleation barriers. Assuming that the free energy of an edge is simply proportional to its height, we obtain $\gamma_{0} / h_{0}=\gamma / h=\gamma_{1}$. If we assume that the edges are bunches of atomic steps, then $\gamma_{1}$ should be the sum of the free energy of an atomic step $\gamma_{\text {step }}$, and the free energy $\gamma_{\text {int }}$ of step-step interactions per atomic step, which is here of entropic origin. ${ }^{1}$ Using standard results of the literature [37], we have $\gamma_{\text {int }}=(\pi k T)^{2} /\left(6 \gamma_{\text {step }} \ell^{2}\right)$ where $\ell$ is the distance between steps. Assuming a tight bunch of steps with $\ell \approx 1$, we find $\gamma_{\text {int }} \approx 0.6$. Summing the two contributions, we obtain $\gamma_{1} \approx \gamma_{\text {step }}+\gamma_{\text {int }} \approx 1$. In contrast, if the distance between steps is large enough, the interaction term is negligible and we obtain $\gamma_{1} \approx \gamma_{\text {step }} \approx 0.4$.

We may now consider the direct formation of a full hole of depth $h=3$ using Eqs. (9) and (10). In the most favorable case $\gamma_{1}=0.42$, we find $R_{c}=1.7, E_{c}=6.6$, and $\exp \left[-E_{c} / T\right] \sim$ $6 \times 10^{-8}$. In the least favorable case $\gamma_{1}=1$, we obtain $R_{c}=4$, $E_{c}=38$, and $\exp \left[-E_{c} / T\right] \sim 6 \times 10^{-41}$. We may therefore conclude that this nucleation process is slower than the layerby-layer scenario described above. Therefore, the formation of holes behind the rim must proceed via a layer-by-layer pealing process. In the simulations, we indeed observe the layer-by-layer formation process of the hole behind the rim, as shown in Fig. 4(b).

In the case where no magic height is present $E_{*}=0$, the layer-by-layer nucleation process is impossible because there is no energy gain to withdraw an atom from the film surface. This is consistent with the observation in Ref. [20] that no hole forms behind the rims in the absence of magic-height thickness.

\section{Incomplete rim closure}

In regime II for small $E_{S}$ and $E_{*}$, we observed that a rim of height $h_{1}$, with $h<h_{1}<h_{*}$ first forms along the film edge. This rim is continuous and extends everywhere along the film edge. Then, on the top of the rim of height $h_{1}$, magic height islands nucleate. These magic-height islands invade the whole rim by means of a zipping mechanism. This process shares similarities with the layer-by-layer increase of the rim height discussed in Ref. [20] in the absence of a magic thickness. In this latter process, a monolayer island of height $h_{1}+1$ invades the rim top facet of height $h_{1}$. However an important difference is seen between the spreading of a monolayer and the spreading of magic height islands.

In order to discuss this difference, let us evaluate the local chemical potentials which enter into play at different edges,

\footnotetext{
${ }^{1} \mathrm{~A}$ better approximation would be to assume that $\gamma=h \gamma_{\text {step }}+$ $(h-1) \gamma_{\text {int }}$, which correctly accounts for the case $h=1$ where there is no interaction term. An even better description of a bunch of steps would also account for the full step density profile. However, our quantitative analysis being very rough, these refinements are beyond the scope of the present paper.
} 

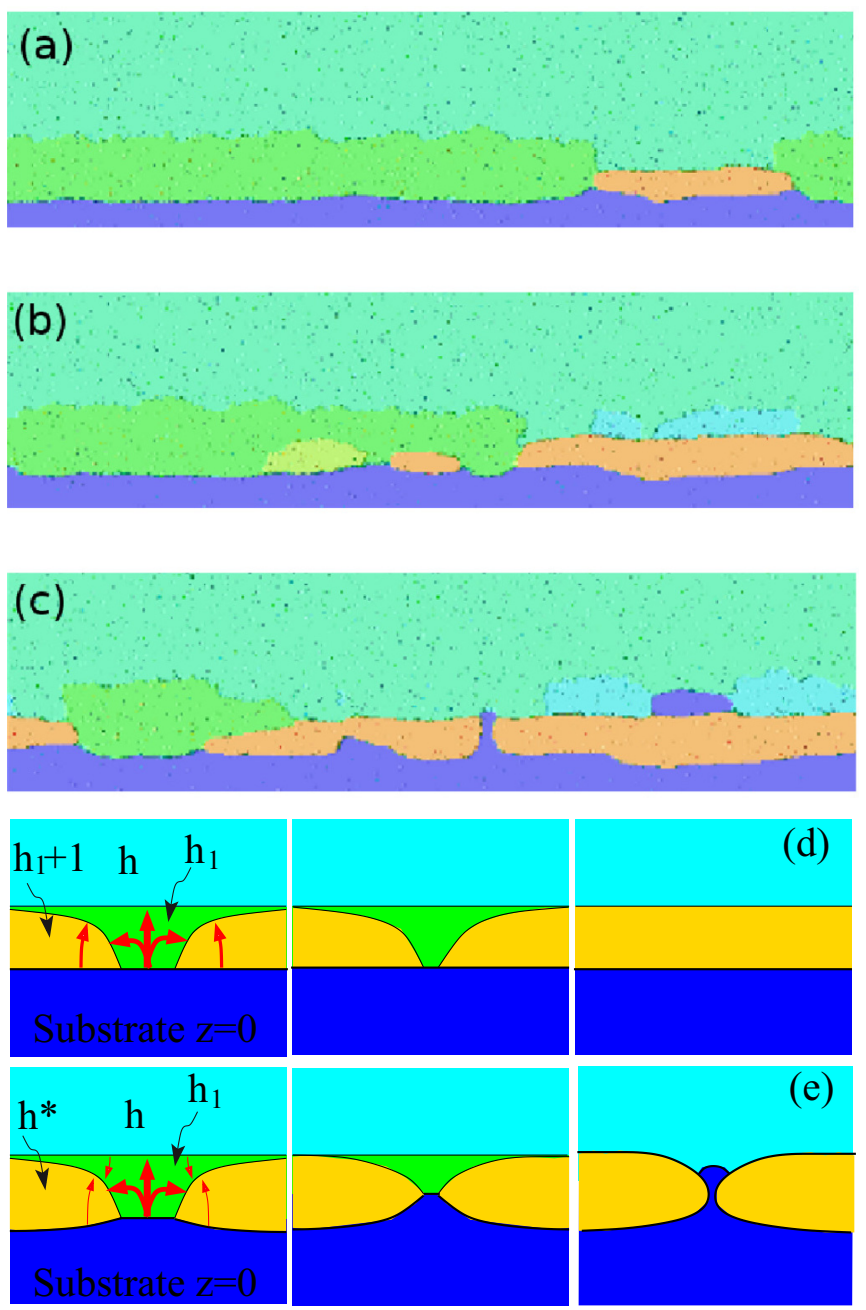

(e)

FIG. 4. (Color online) Details of the rim dynamics observed in KMC in regime II (small $E_{S}$ and $E_{*}$ ). Parameters: size $800 \times 800$, $T=0.4, h=3, E_{S}=0.4, h_{*}=7 E_{*}=-0.5$. (a) First, a rim of height $h_{1}$ forms, with $h<h_{1}<h_{*}$ (in green), with a process similar to that described in Ref. [20]. Then, a magic-height rim (orange) is nucleated and extends along the dewetting front by means of a zipping mechanism. As a consequence of mass conservation, the width of the magic-height rim is smaller than that of the initial rim. (b) Another magic-height zone is nucleated along the rim. In addition, the layer-by-layer nucleation of a hole behind the rim is observed on the right side. (c) In the center of the image, we observe that the expanding magic-height rims do not merge when they approach each other: This is the incomplete rim closure process discussed in the text. On the right side, the layer-by-layer nucleation of a hole proceeds and has reached the substrate. In (d) and (e), we present a schematic top view to discuss the incomplete rim closure mechanism. (d) and (e) represent, respectively, the cases without and with a magic height.

such as the film edge, the edge of a monolayer, or the edge of a magic island. We postpone the discussion of the influence of edge curvature effects to Sec. III D. As shown in Ref. [35], the chemical potential at an edge of height $\Delta z$ is then simply $\Delta E / \Delta z$, where $\Delta E$ is the energy cost per unit area when moving this edge.

We start with the case of dewetting without a magic height, during the spreading of a monolayer on the rim facet, as shown in Fig. 4(d). At the dewetting front between $z=0$ and $z=h_{1}$, we have $\mu_{0 \mid h_{1}}=E_{S} / h_{1}$, and at the dewetting front between $z=0$ and $z=h_{1}+1$, we have $\mu_{0 \mid h_{1}+1}=E_{S} /\left(h_{1}+1\right)$. In addition, at the edge of the monolayer on the rim facet $\mu_{h_{1} \mid h_{1}+1} \approx 0$, and similarly at the frontier between the edge of the rim facet and the film $\mu_{h_{1} \mid h} \approx 0$. Thus, the driving forces for the mass fluxes toward the edge of the monolayer are $\mu_{0 \mid h_{1}}-\mu_{h_{1} \mid h_{1}+1}=E_{S} / h_{1}$ from the lower side and $\mu_{0 \mid h_{1}+1}-$ $\mu_{h_{1} \mid h_{1}+1}=E_{S} /\left(h_{1}+1\right)$ from the upper side. These driving forces are not very different, and as a consequence the related mass fluxes are of the same order of magnitude and the atomic step on the top facet is therefore fed from both sides rather symmetrically.

In contrast, for the spreading of a magic-height rim on the rim facet shown in the schematics of Fig. 4(e), the chemical potentials are $\mu_{0 \mid h_{1}}=E_{S} / h_{1}, \mu_{0 \mid h^{*}}=\left(E_{S}+E_{*}\right) / h^{*}, \mu_{h^{*} \mid h_{1}}=$ $E_{*} /\left(h^{*}-h_{1}\right)$, and $\mu_{h 1 \mid h} \approx 0$. As a consequence, the mass fluxes toward the edge of the magic island on the rim facet are now proportional to $\mu_{0 \mid h_{1}}-\mu_{h^{*} \mid h_{1}}=E_{S} / h_{1}-E_{*} /\left(h^{*}-h_{1}\right)$ from the lower side and to $\mu_{0 \mid h^{*}}-\mu_{h^{*} \mid h_{1}}=\left(E_{S}+E_{*}\right) / h^{*}-$ $E_{*} /\left(h^{*}-h_{1}\right)$ from the upper side. Since $h_{1}<h_{*}$ and $E_{S}>$ $E_{S}+E_{*}$, one has $\mu_{0 \mid h_{1}}-\mu_{h^{*} \mid h_{1}}>\mu_{0 \mid h^{*}}-\mu_{h^{*} \mid h_{1}}$. As a consequence, the mass flux from the edge of the film of height $h_{1}$ is now significantly larger than the mass flux coming from the edge of height $h_{*}$.

As a summary, we have seen that the mass fluxes toward the edge of a monolayer spreading on a facet are essentially symmetric, while the edge of a magic island is essentially fed from the lower side. As a consequence, when two monolayer islands spread toward each other as in Fig. 4(d), they are both still fed from the upper side when getting close to each other. Hence, they are not significantly slowed down when the distance between them gets smaller, and they finally collide and merge. However, when two magic-height islands spread and get close to each other as shown in Fig. 4(e), they are mainly fed by the rim-substrate edge between them. As shown in Fig. 4(e), this leads to an increased motion of this part of the rimsubstrate edge. But since $h_{1}<h_{*}$, from mass conservation the two magic islands simply cannot meet if they are fed only by the rim of height $h_{1}$. Hence, the rim-substrate edge between the magic islands moves beyond the magic islands leaving a gap.

Ultimately, the dewetting proceeds through this gap toward the film behind the rim. However, due to statistical fluctuations the dynamics is not deterministic and magic height rims may still merge from time to time, as seen in Fig. 4.

\section{Maximum velocity principle}

We now propose a model to predict the typical wavelength and dewetting velocity of a dewetting front. We assume that this dewetting front is composed by an alternation of fingers of height $h_{*}$ and width $\lambda_{*}$, and void-fingers of height $h=0$ and width $\lambda_{0}$. By construction, the wavelength $\lambda$ of the front pattern is $\lambda=\lambda_{0}+\lambda_{*}$. In addition, from mass conservation $h \lambda=h_{*} \lambda_{*}$. As a consequence

$$
\begin{gathered}
\lambda_{*}=\frac{h}{h_{*}} \lambda \\
\lambda_{0}=\frac{h_{*}-h}{h_{*}} \lambda .
\end{gathered}
$$


We now aim to describe the driving force by which atoms in the film form fingers. The chemical potential at the fronts is expected to be [35]

$$
\begin{gathered}
\frac{\mu_{0}}{T}=\frac{E_{S}}{h T}+\Gamma \kappa_{0} \\
\frac{\mu_{*}}{T}=\frac{E_{*}}{\left(h_{*}-h\right) T}+\Gamma_{*} \kappa_{*},
\end{gathered}
$$

where $\kappa_{0}$ and $\kappa_{*}$ are the edge curvatures. We have also defined $\Gamma=\gamma /(h T)$ and $\Gamma_{*}=\gamma_{*} /\left(h_{*} T\right)$, where $\gamma$ and $\gamma_{*}$ are the free energies of the edge of a film of height $h$ or $h_{*}$ respectively.

Assuming fixed contact angles at the triple points between the film-substrate, film-magic-island, and substrate-magicisland edge

$$
\begin{gathered}
\kappa_{0}=\frac{-2 \alpha}{\lambda_{0}}, \\
\kappa_{*}=\frac{2 \alpha_{*}}{\lambda_{*}},
\end{gathered}
$$

where $\alpha$ and $\alpha_{*}$ are numbers accounting for the edge geometry. We now write the velocity of the void-fingers as resulting from diffusion limited dynamics

$$
V=G \frac{1}{h} \frac{D c_{e q}}{\lambda}\left(\mathrm{e}^{\mu_{0} / T}-\mathrm{e}^{\mu_{*} / T}\right),
$$

where $G$ is a dimensionless number, which accounts for geometric effects. We assume that $\Gamma, \Gamma_{*} \ll \lambda$. We also assume that the free energy of a film edge is proportional to its height, $\gamma / h=\gamma_{*} / h=\gamma_{1}$. This last assumption suggests that, following the Young contact angle relation, tangential matching should be observed between the film-substrate and the film-magic-island edges at the triple point. As a consequence, we expect $\alpha \approx \alpha_{*} \approx 1$. Then we obtain

$$
V=G \frac{1}{h} \frac{D c_{e q}}{\lambda}\left(\Delta \mathrm{e}-\frac{2 \Gamma}{\lambda} \Sigma \mathrm{e}\right),
$$

where we have defined

$$
\begin{aligned}
\Delta \mathrm{e} & =\exp \left[\frac{E_{S}}{T h}\right]-\exp \left[\frac{E_{*}}{T\left(h_{*}-h\right)}\right], \\
\Sigma \mathrm{e} & =\frac{h_{*}}{h_{*}-h} \exp \left[\frac{E_{S}}{T h}\right]+\frac{h_{*}}{h} \exp \left[\frac{E_{*}}{T(h *-h)}\right] .
\end{aligned}
$$

Maximizing Eq. (18) with respect to $\lambda$, one finds

$$
\begin{gathered}
\frac{\lambda}{\Gamma}=\frac{4 \Sigma \mathrm{e}}{\Delta \mathrm{e}} . \\
V=G \frac{1}{h} \frac{D c_{e q}}{\Gamma} \frac{(\Delta \mathrm{e})^{2}}{8 \Sigma \mathrm{e}} .
\end{gathered}
$$

Following the same lines, the velocity $V_{*}$ of the magic height fingers is found to obey a similar law

$$
V_{*}=G_{*} \frac{1}{h_{*}-h} \frac{D c_{e q}}{\Gamma} \frac{(\Delta \mathrm{e})^{2}}{8 \Sigma \mathrm{e}} .
$$

Since $V=V_{*}$, we must require

$$
\frac{G_{*}}{h_{*}-h}=\frac{G}{h}=g,
$$

where the constant $g$ is expected to be a symmetric function of $h$ and $h_{*}-h$.

We now compare the model results with KMC simulations. From Eqs. (20) and (23), we expect that

$$
\begin{gathered}
\lambda \frac{\Delta \mathrm{e}}{\Sigma \mathrm{e}}=4 \Gamma \\
V \frac{\Sigma \mathrm{e}}{(\Delta \mathrm{e})^{2}}=g \frac{D c_{e q}}{8 \Gamma}
\end{gathered}
$$

are constant.

In order to check the results of the model, we have measured the size $\lambda$ and velocity $V$ in the KMC simulations. The measured velocity $V$ is the global velocity of the dewetting front, which is expected to be the same as the finger velocity in regime I when the fingers are orthogonal to the front, but not in regime II, where the fingers evolve in a more complex fashion. However, as shown in Fig. 5(a), KMC simulations provide reasonable data collapse for $V$, and a good one for $\lambda$. The value of $\lambda$ is almost constant for $E_{S} \geqslant 0.5$, but increases
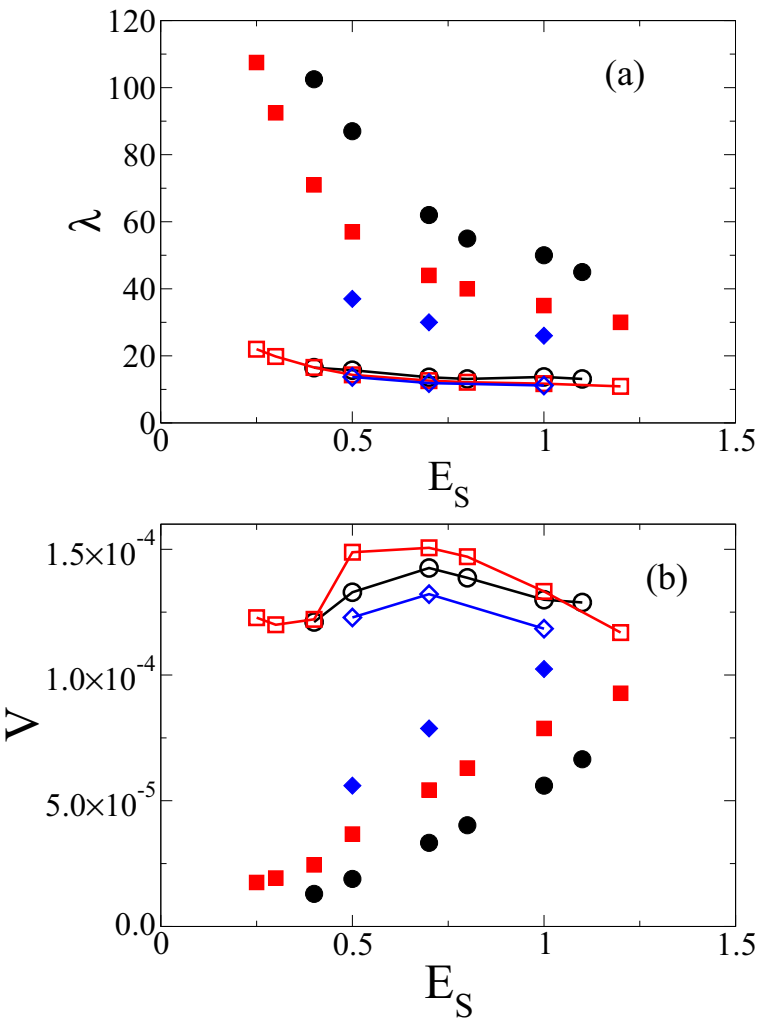

FIG. 5. (Color online) Maximum velocity principle. (a) Wavelength $\lambda$ of the final pattern composed only by magical height islands and (b) dewetting front velocity $V$. Nonconnected full symbols are results from simulations. Open symbols connected by solid lines correspond to the expressions $\lambda \Delta e / \Sigma e$ and $V \Sigma e /(\Delta e)^{2}$ appearing in Eqs. (24) and (25). The maximum velocity principle discussed inn Sec. III D predicts that these quantities are constant. Parameters: system size $800 \times 800, h=3, T=0.4, h_{*}=7$. Symbols: black circles: $E_{*}=-0.5$, red squares $E_{*}=-1$, and blue diamonds $E_{*}=-2$. 
slightly for small $E_{S}$. From the $\mathrm{KMC}$ results, we obtain

$$
\begin{gathered}
\lambda \frac{\Delta \mathrm{e}}{\Sigma \mathrm{e}}=12.5 \pm 2.5 \\
V \frac{\Sigma \mathrm{e}}{(\Delta \mathrm{e})^{2}}=(1.3 \pm 0.1) \times 10^{-4}
\end{gathered}
$$

leading to

$$
\Gamma=3.1 \pm 0.6 .
$$

Such a value of $\Gamma$ is in reasonable agreement with the results of Sec. III B. Indeed, since $\Gamma=\Gamma_{*}=\gamma_{1} / T$, using the value of $\Gamma \approx 3$ obtained above, we find $\gamma_{1}=T \Gamma \approx 1.2$ in good agreement with the theoretical value $\gamma_{1} \approx 1$ proposed in Sec. III B.

Finally, our results also allow one to evaluate the geometrical parameter $g$. In order to do so, we first need to determine $D$ and $c_{e q}$. Using the results of Ref. [33], we have $D=1 / 4$, and $c_{e q}=\mathrm{e}^{-2 / T}$, leading to

$$
g=1.9 \pm 0.5 .
$$

Such an order of magnitude of $g$ seems reasonable, because it is of the order of one, as expected for a geometrical prefactor.

\section{HOMOGENEOUS NUCLEATION FOR A CONTINUOUS FILM}

In order to discuss the homogeneous nucleation of holes in this section, we focus on the dynamics of an initially continuous film (with no edge). In experimental systems, the dewetting process of continuous films is often nucleated at defects $[4,11]$. These defects can be pre-existing holes, impurities, grain boundaries, or film edges. However, in very thin films with several layers only, homogeneous nucleation of holes can be triggered by thermal fluctuations [20]. An example of homogeneous nucleation may have been identified during the dewetting of very thin $\mathrm{Si}(111)$ films with thicknesses smaller than $4 \mathrm{~nm}$ on $\mathrm{SiO}_{2}$ substrates [7]. For extremely thin metallic films, we therefore also expect homogeneous nucleation to be relevant, with a possibly nontrivial coupling to magic thicknesses.

In order to study these effects, we perform simulations starting from a continuous film of thickness $h=3$. We have performed two sets of simulations: (i) with $h_{*}=7$ and (ii) with $h_{*}=5$.

Several observations are in order. First, we notice that islands organize in starlike structures centered around the nucleation centers in case (ii), while the finger structure is less organized in case (i), as seen from Figs. 6 and 7.

A second striking difference between cases (i) and (ii) is that islands form a percolating network in case (ii) with $h_{*}=5$, while they do not percolate in case (i) with $h_{*}=7$. This can be traced back to the final coverage $\theta_{*}$ fixed by mass conservation $\theta_{*}=h / h_{*}$, which is equal to $\theta_{*}=0.6$ in case (i) and to $\theta_{*}=0.43$ in case (ii). A percolation transition is roughly expected for $\theta_{*} \approx 1 / 2$, although the precise value of the transition depends on the details of the spatial organization of the islands.

Third, the nucleation pathway is different in cases (i) and (ii). The nucleation process starts with the formation of small
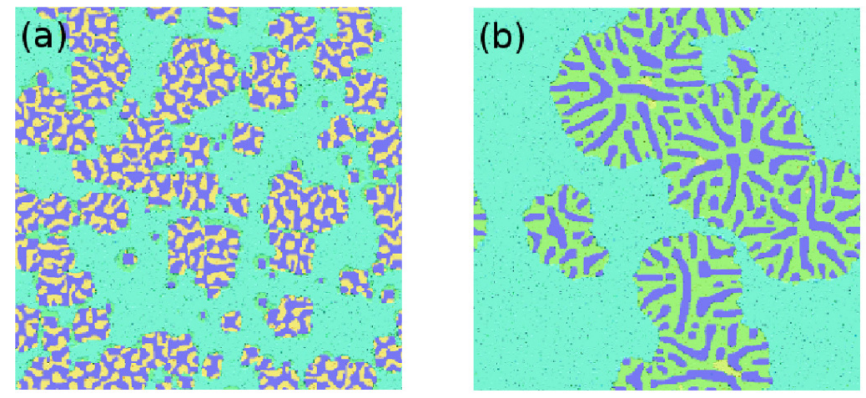

\section{(c)}

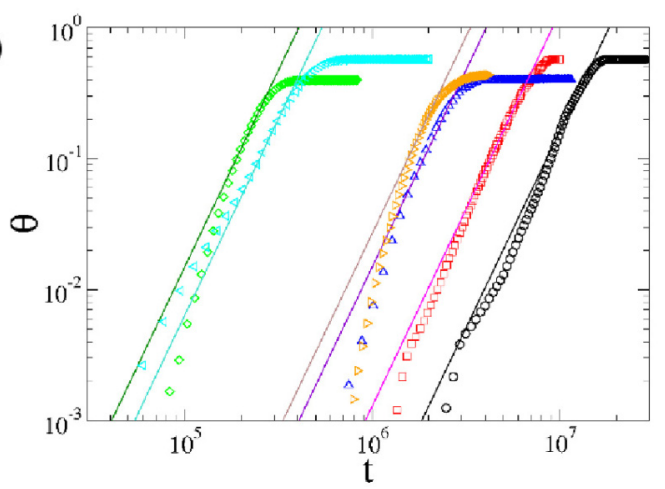

FIG. 6. (Color online) Homogeneous nucleation. We started with a continuous film of thickness $h=3$, in a system of size $800 \times 800$, with $T=0.4$. (a) $h_{*}=7, E_{S}=2$, and $E_{*}=-2$; (b) $h_{*}=5, E_{S}=1$, and $E_{*}=-0.5$. (c) Uncoverage $\theta$ during homogeneous dewetting as a function of time. Solid lines are guides to the eye $\sim t^{3}$. Symbols represent KMC simulation results with $T=0.4, h=3$, and $\left(E_{S}\right.$, $\left.E_{*}, h *\right): \bigcirc(0.3,-2.0,7) ; \square(0.5,-2.0,7) ; \diamond(1.0,-1.0,5) ; \triangleleft$ $(2.0,-2.0,7) ; \triangle(0.7,-0.5,7) ; \triangleright(1.0,-0.5,5) ; \triangleleft$ correspond to the parameters of (a) and $\triangleright$ correspond to the parameters of (b).

holes in case (i) with $h_{*}=7$. Subsequently, magic-height islands form around the holes. In contrast, the nucleation process starts with magic-height islands on the surface of the film in case (ii) with $h_{*}=5$. Then, holes form in the vicinity of these magic islands with a process similar to the layer-by-layer nucleation scenario described in Sec. III B.

It is tempting to speculate that the differences for island percolation and for the nucleation process could be observed in general as a function of the relative values of $h_{*}$ and $h$ : nonpercolating islands and initial hole formation for $h_{*}-h>h$, and percolating islands and initial magic-island formation for $h_{*}-h<h$.

Finally, the substrate area uncovered by the dewetting process grows as $t^{3}$, as shown in Fig. 6(c). This is similar to the behavior observed in the dewetting of monolayer films [33], but different from homogeneous nucleation in (multilayer) ultrathin films without magic height, where the approximate scaling $\sim t^{2}[20,34]$ was observed. As discussed in Ref. [20], the $t^{2}$ behavior is a consequence of the thickening of the dewetting rim, which slows down the opening of holes leading to a front velocity with the approximate scaling $t^{-1 / 2}$. In contrast, the dewetting front velocity is constant in the absence of a stable dewetting rim, as observed for monolayer islands [33], or for thin films with a magic height, leading to $t^{3}$. 

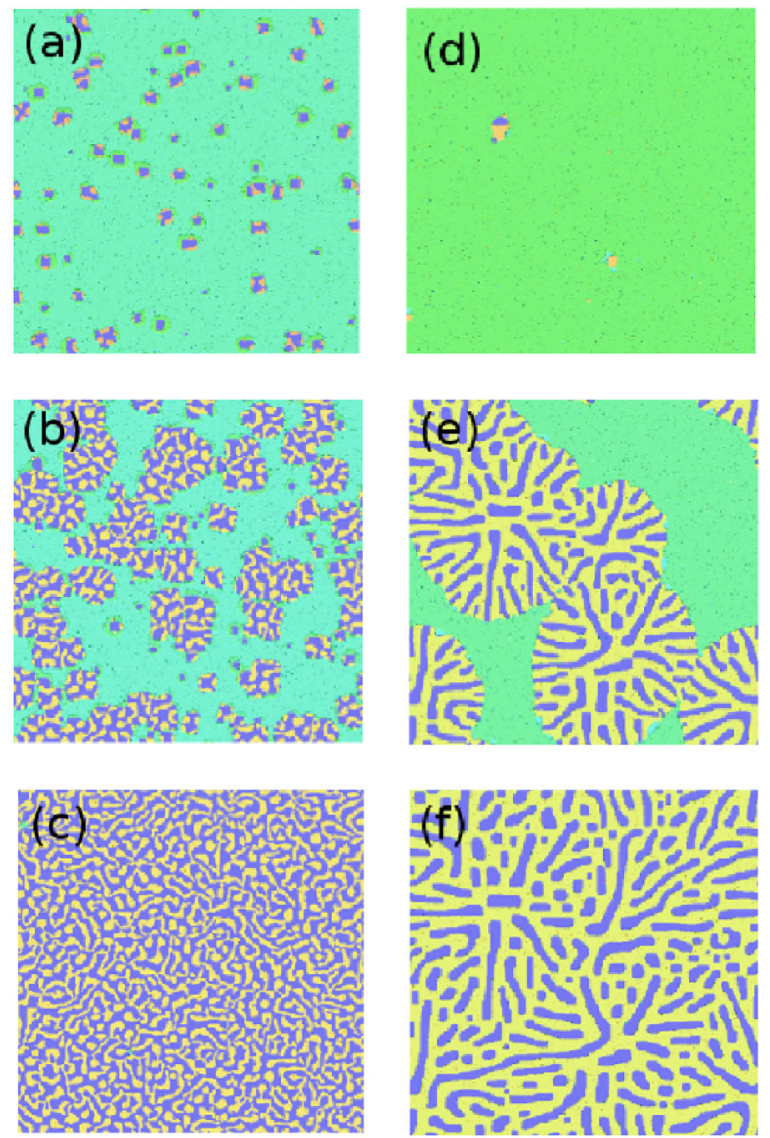

FIG. 7. (Color online) Dewetting process during homogeneous dewetting with an initial film thickness $h=3$, and $T=0.4$ in a $800 \times 800$ system. (a)-(c) correspond to $h_{*}=7, E_{S}=2$, and $E_{*}=-2$. Holes are nucleated initially before magic-height islands in (a). The final state is a nonpercolating network of islands. (d)-(f) correspond to $h_{*}=5, E_{S}=0.7$, and $E *=-0.5$. Magic-height islands are formed before holes in the initial stages (d). The final state exhibits a percolating network of magic-size islands.

\section{COMPARISON WITH EXPERIMENTS}

While our simplified model does not provide a complete description of a given experimental system in all its details, we claim that it can help identify the specific features of electronic dewetting in experiments and that it provides quantitative predictions that are consistent with some experimental results. When $h=3$ and $h_{*}=5$, the magic-height flat top islands resulting from the dewetting process form a network [see Fig. 7(f)], similar to the observations of Thürmer et al. [2] and Gavioli et al. [29] for Ag/Si. In Ref. [2] Ag is deposited at room temperature where $\mathrm{Ag}$ is mobile at the surface, so that the experimental conditions mix growth and dewetting. However in Ref. [29], deposition is performed at low temperatures, and the film is annealed at room temperature after the deposition process. In both cases, the experiments lead to similar wormlike structures with a lateral length scale $\sim 20 \mathrm{~nm}$. Note that the average film thickness is 2.2 ML in Ref. [29] and $\sim 3 \mathrm{ML}$ in Ref. [2].

Using the surface free energy [38] of $\mathrm{Ag}(111)$ is $\sigma=$ $76 \mathrm{meV}^{-2}$, Thürmer et al. estimated a dewetting energy $E_{S}$ to be of the order of $\mathrm{meV}$ to tens of $\mathrm{meV} / \AA^{-2}$. We here assume $E_{S} \sim 50 \mathrm{meV}^{-2}$. Using Fig. 2 of Z. Zhang et al. [25], we find for $\mathrm{Ag}: E_{*} \sim 0.02 \mathrm{meV}^{-2}$. In addition, the magic thickness of $\mathrm{Ag} / \mathrm{Si}$ was observed experimentally [28,32]. For this system, Hirayama [32] proposed $h_{*} \approx 6$ monolayers. A simple estimate of the free energy of the film edge is based on the surface energy of the top facet of the film $\sigma=76 \mathrm{meV} \AA^{-2}$. With these assumptions and using Eq. (26), we obtain $\lambda \approx$ $30 \mathrm{~nm}$ in reasonable agreement with the experiments. For $\mathrm{Ag} / \mathrm{Si}$ elastic effects could also play an important role [28], but our analysis based solely on electronic effects seems to catch the correct order of magnitude of $\lambda$.

Another feature which appears in experimental systems, but which is not accounted for in our model, is the presence of multiple magic heights [25]. We have used a model based on experimental observations, where the most relevant and striking feature of the film morphology, i.e., the wormlike structure, is a consequence of the smallest magic height. This is rooted in two facts.

First, the energy gain related to magic heights usually decreases rapidly with the film thickness [25], so that the consequences of larger magic heights tend to be weaker and are much more difficult to observe. Second, the experiments discussed here report on the deposition of a small amount of mass, leading to an average film thickness smaller than the first magic height. Our model applies to such cases, at least in the first stages of the dynamics, where only the first magic height is reached. However, in the case of $\mathrm{Ag} / \mathrm{Si}(111)$ beyond the initial stages of the dewetting process, the film is further destabilized during annealing and slowly reaches larger magic heights, as reported in Ref. [2]. Since the magic height energies decrease rapidly with increasing film thicknesses, three-dimensional islands form in the final stages. This full process cannot be described within our model with a single magic height.

In addition, our results could provide some insights about the initial dewetting behavior of thicker films in the presence of multiple magic heights. Indeed, let us consider a system with an ensemble of magic heights $h_{* n}$, with energies $E_{* n}$, where $n=1,2, \ldots$. We then start with a film of height $h_{\text {new }}$ such that $h_{* m}<h_{\text {new }}<h_{* m+1}$, this film will decompose into parts of height $h_{* m}$, and parts with height $h_{* m+1}$ to gain energy. Such a process is actually similar to the case discussed in our model, and the results discussed in Sec. IV apply with the following substitutions: $h \rightarrow h_{\text {new }}-h_{* m}, h_{*} \rightarrow h_{* m+1}-h_{* m}, E_{S} \rightarrow$ $-E_{* m}$, and $E_{*} \rightarrow E_{* m+1}$. However, once again, late stages of the dewetting process, where other magic thicknesses may come into play, cannot be accounted for within our model.

\section{CONCLUSIONS}

We have analyzed the dewetting of a thin solid film, with initial thickness $h$, when a magic thickness $h_{*}$ is energetically favored. We considered the case where $h_{*}$ is of the same order of magnitude as $h$, which corresponds to the experimental observations in ultrathin metallic films. Using KMC simulations, the dewetting dynamics is observed until a pattern composed only by magic-height islands appears.

In the case where the driving forces for dewetting are small enough for dewetting to proceed from the edges of the film, we 
find two regimes. In regime I, for large enough driving forces, magic-height fingers are perpendicular to the dewetting front. In regime II, for lower driving forces, magic height fingers are globally parallel to the dewetting front. The morphology with magic-height fingers parallel to the front in regime II shares similarities with that resulting from the periodic mass shedding scenario proposed by Wong et al. [18]. However, the origin of this geometry is different, and can be traced back to the induced layer-by-layer nucleation of holes behind the rim. Furthermore, magic-height dewetting rims exhibits an other kind of instability: incomplete rim closure.

In both regimes, the size of the islands and velocity of the front observed in simulations are in agreement with a simple analytical model based on a maximum velocity principle. For large enough driving forces, homogeneous nucleation of holes is observed in a continuous film. Two regimes are identified depending on the relative values of $h$ and $h_{*}$. For small values of $h_{*}$ (corresponding typically to $h_{*}-h<h$ ), the dewetting process is initiated by the formation of magic-height islands, and leads to the formation of a percolating network of islands. In contrast, for large values of $h_{*}$ (i.e., $h_{*}-h>$ $h)$, the dewetting process is initiated by the nucleation of holes, and leads to a nonpercolating network of magic-height islands.

The morphology and the order of magnitude of the magic-height finger width are in agreement with experimental observations, suggesting that we have caught the relevant physical ingredients of these systems. However, much yet remains to be done in order to reach a complete and quantitative understanding of the complex dynamics of electronic dewetting.

\section{ACKNOWLEDGMENTS}

We acknowledge support from Ciência sem Fronteiras/CNPq Grant No. PVE 400578/2012-3, and OPL acknowledges support from ANR-12-BS04-0008 (LOTUS).
[1] E. Jiran and C. Thompson, Thin Solid Films 208, 23 (1992).

[2] K. Thürmer, E. D. Williams, and J. E. Reutt-Robey, Phys. Rev. B 68, 155423 (2003).

[3] B. Krause, A. C. Drr, F. Schreiber, H. Dosch, and O. H. Seeck, J. Chem. Phys. 119, 3429 (2003).

[4] B. Yang, P. Zhang, D. E. Savage, M. G. Lagally, G.-H. Lu, M. Huang, and F. Liu, Phys. Rev. B 72, 235413 (2005).

[5] M. Coll, J. Gázquez, A. Pomar, T. Puig, F. Sandiumenge, and X. Obradors, Phys. Rev. B 73, 075420 (2006).

[6] E. Dornel, J.-C. Barbé, F. de Crécy, G. Lacolle, and J. Eymery, Phys. Rev. B 73, 115427 (2006).

[7] Z. A. Burhanudin, R. Nuryadi, Y. Ishikawa, and M. Tabe, Thin Solid Films 508, 235 (2006).

[8] K. F. McCarty, J. C. Hamilton, Y. Sato, A. Saá, R. Stumpf, J. de la Figuera, K. Thürmer, F. Jones, A. K. Schmid, A. A. Talin, and N. C. Bartelt, New J. Phys. 11, 043001 (2009).

[9] E. J. Luber, B. C. Olsen, C. Ophus, and D. Mitlin, Phys. Rev. B 82, 085407 (2010).

[10] H. Galinski, T. Ryll, P. Elser, J. L. M. Rupp, A. Bieberle-Hütter, and L. J. Gauckler, Phys. Rev. B 82, 235415 (2010).

[11] E. Bussmann, F. Cheynis, F. Leroy, P. Müller, and O. PierreLouis, New J. Phys. 13, 043017 (2011).

[12] F. Cheynis, E. Bussmann, F. Leroy, T. Passanante, and P. Müller, Phys. Rev. B 84, 245439 (2011).

[13] J. Ye and C. V. Thompson, Acta Mater. 59, 582 (2011).

[14] C. V. Thompson, Annu. Rev. Matter. Res. 42, 399 (2012).

[15] F. Leroy, F. Cheynis, T. Passanante, and P. Müller, Phys. Rev. B 85, 195414 (2012).

[16] M. Aouassa, L. Favre, A. Ronda, H. Maaref, and I. Berbezier, New J. Phys. 14, 063038 (2012).

[17] D. Srolovitz and S. Safran, J. Appl. Phys. 60, 255 (1986).

[18] H. Wong, P. Voorhees, M. Miksis, and S. Davis, Acta Mater 48, 1719 (2000).
[19] W. Kan and H. Wong, J. Appl. Phys. 97, 043515 (2005).

[20] O. Pierre-Louis, A. Chame, and Y. Saito, Phys. Rev. Lett. 103, 195501 (2009).

[21] M. Dufay and O. Pierre-Louis, Phys. Rev. Lett. 106, 105506 (2011).

[22] A. Chame and O. Pierre-Louis, Phys. Rev. E 85, 011602 (2012).

[23] F. K. Schulte, Surf. Sci. 55, 427 (1976).

[24] P. J. Feibelman, Phys. Rev. B 27, 1991 (1983).

[25] Z. Zhang, Q. Niu, and C.-K. Shih, Phys. Rev. Lett. 80, 5381 (1998).

[26] Y. Jia, B. Wu, C. Li, T. L. Einstein, H. H. Weitering, and Z. Zhang, Phys. Rev. Lett. 105, 066101 (2010).

[27] B. J. Hinch, C. Koziol, J. Toennies, and G. Zhang, Europhys. Lett. 10, 341 (1989).

[28] L. Huang, S. Jay Chey, and J. H. Weaver, Surf. Sci. 416, L1101 (1998).

[29] L. Gavioli, K. R. Kimberlin, M. C. Tringides, J. F. Wendelken, and Z. Zhang, Phys. Rev. Lett. 82, 129 (1999).

[30] P. Czoschke, H. Hong, L. Basile, and T.-C. Chiang, Phys. Rev. Lett. 91, 226801 (2003).

[31] M. Miyazaki and H. Hirayama, Surf. Sci. 602, 276 (2008).

[32] H. Hirayama, Surf. Sci. 603, 1492 (2009).

[33] O. Pierre-Louis, A. Chame, and Y. Saito, Phys. Rev. Lett. 99, 136101 (2007).

[34] A. Chame and O. Pierre-Louis, C. R. Phys. 14, 553 (2013).

[35] O. Pierre-Louis, A. Chame, and M. Dufay, Eur. Phys. J. B 77, 57 (2010).

[36] P. G. de Gennes, Rev. Mod. Phys. 57, 827 (1985).

[37] C. Misbah, O. Pierre-Louis, and Y. Saito, Rev. Mod. Phys. 82, 981 (2010).

[38] M. Methfessel, D. Hennig, and M. Scheffler, Phys. Rev. B 46, 4816 (1992). 